\title{
Genus Two Hyperelliptic Curve Coprocessor
}

\author{
N. Boston, T. Clancy, Y. Liow, and J. Webster \\ Illinois Center for Cryptography and Information Protection* \\ Coordinated Science Laboratory, University of Illinois, Urbana-Champaign \\ 1308 West Main Street, Urbana, IL 61801 \\ \{n-boston, tclancy, liow, jewebste\}@uiuc.edu
}

\begin{abstract}
Hyperelliptic curve cryptography with genus larger than one has not been seriously considered for cryptographic purposes because many existing implementations are significantly slower than elliptic curve versions with the same level of security. In this paper, the first ever complete hardware implementation of a hyperelliptic curve coprocessor is described. This coprocessor is designed for genus two curves over $\mathbb{F}_{2^{113}}$. Additionally, a modification to the Extended Euclidean Algorithm is presented for the GCD calculation required by Cantor's algorithm. On average, this new method computes the GCD in one-fourth the time required by the Extended Euclidean Algorithm.
\end{abstract}

\section{Introduction}

Hyperelliptic curves (HEC) are a generalization of elliptic curves and the first suggestion of their cryptographic use was made by N. Koblitz at Crypto '88 ([10]). Elliptic curve cryptography (ECC) has received much attention because it offers several benefits over other public-key cryptosystems, such as RSA. With a higher security per bit than RSA, ECC allows for a comparable level of security with a smaller key size. Additionally, many have reported ECC hardware implementations require significantly fewer transistors.

This paper presents concrete performance results from a hardware-based genus two hyperelliptic curve coprocessor over $\mathbb{F}_{2^{113}}$. Additionally, these performance characteristics are compared to a software implementation over the same field and curve.

The hardware version was implemented on a Field Programmable Gate Array (FPGA). FPGAs allow programmers to input a logic structure which will be emulated using the extensive set of gates available on the FPGA. These logic structures are created using Hardware Description Language (HDL). In this implementation, Verilog, a popular HDL, was used to describe the hardware. From there, the Xilinx Integrated Software Environment was used to synthesize and implement the logic design for a Xilinx Virtex II FPGA. Additionally, the Modeltech Microsim simulator was used to verify the correctness of the design.

\footnotetext{
* Research supported by NSF EIA 00-88063
} 
A software version written in $\mathrm{C}++$ was compiled using Microsoft's Visual Studio and tested on a Pentium system. Further results on the software implementation will be available in a forthcoming publication.

While no other complete hardware-based HEC coprocessor has been previously completed, 20] presents many of the architectural requirements. 20] did not achieve a space-efficient implementation and consequently no accurate timing and area values were included. In contrast, this work presents a complete implementation with accurate timing and area requirements.

Several theoretic results are also included. An alternate for computing the GCD of three polynomials of small degree is presented, which significantly decreases processing time in Cantor's Algorithm. The paper also presents a bound on the expected computation time of point multiplication using a point adder and a point doubler in parallel.

\section{Basic Algorithms}

This paper assumes the reader possesses a familiarity with groups, rings, and fields, in addition to a basic understanding of elliptic curve cryptography. For a complete review of abstract algebra, see [7] or [5]. For a background on the mathematical concepts involved in elliptic curves, see [17] or [2].

Elements in the ECC group are pairs of finite field elements. The corresponding group based on the hyperelliptic curve is its Jacobian. The points are described by pairs of polynomials over a finite field. For a more rigorous treatment of hyperelliptic curves, see [12].

This section develops the various algorithms used to handle binary operations in finite fields, polynomial rings, and on the Jacobian of the hyperelliptic curve. Additionally, some theoretic results on the processing time of certain algorithms are derived.

\section{$2.1 \quad$ Finite Fields}

Using a polynomial basis over $\mathbb{F}_{2^{n}}$, any finite field element can be represented as coefficients of powers of $x$, which can be conveniently stored in memory as an $n$-bit vector. Another basis called optimal normal basis (ONB) is also commonly used because it allows for very efficient squaring; however, ONB is very slow when performing inversions. This implementation uses polynomial basis.

Field Addition. When adding two field polynomials, their sum is the sum of the coefficients. Under a characteristic two finite field, implementing addition requires bitwise XOR-ing together the two vectors representing the polynomial coefficients.

Field Multiplication. Multiplication can be efficiently achieved by using a slightly modified version of the standard grade-school algorithm. A method for 
reducing the product as the algorithm progresses is required to prevent it from growing too large, and is presented in Algorithm 1. An alternate field multiplication algorithms originally presented in [19] multiplies $D$ bits simultaneously, requiring $\left\lceil\frac{n}{D}\right\rceil$ clock cycles to complete; however, implementing such multipliers in a hyperelliptic environment would not be area efficient enough to fit on most FPGAs.

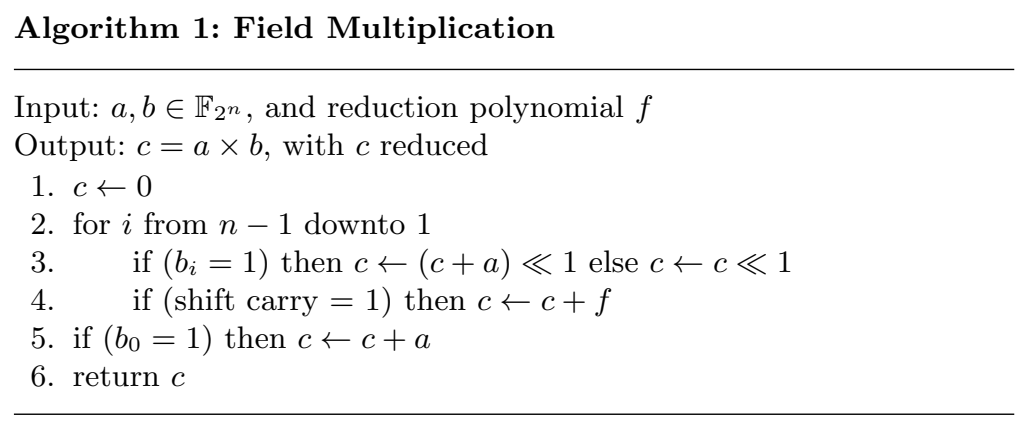

Field Squaring. In a characteristic $p$ finite field, $\left(x_{1}+\cdots+x_{n}\right)^{p}=x_{1}^{p}+\cdots x_{n}^{p}$. Hence when squaring in characteristic two, the powers of the basis terms double, essentially spacing out the bits in the vector representation. For example, $\left(x^{2}+\right.$ $x+1)^{2}=x^{4}+x^{2}+1$. After spacing the bits, the vector is twice its original length, and the higher bits may need to be reduced. Algorithm 2 presents reassignment of the lower bits, and reduction of the upper bits.

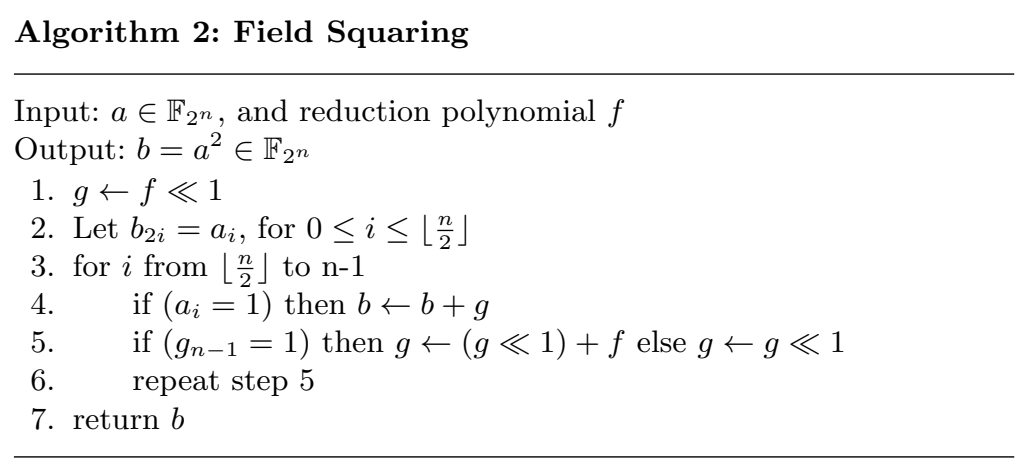

Field Inversion. Inversion of finite field elements uses a modified version of the Extended Euclidean Algorithm as reported in [6]. This version only keeps track 
of the minimal set of required information, and uses bit shifting with XOR. The details are in Algorithm 3.

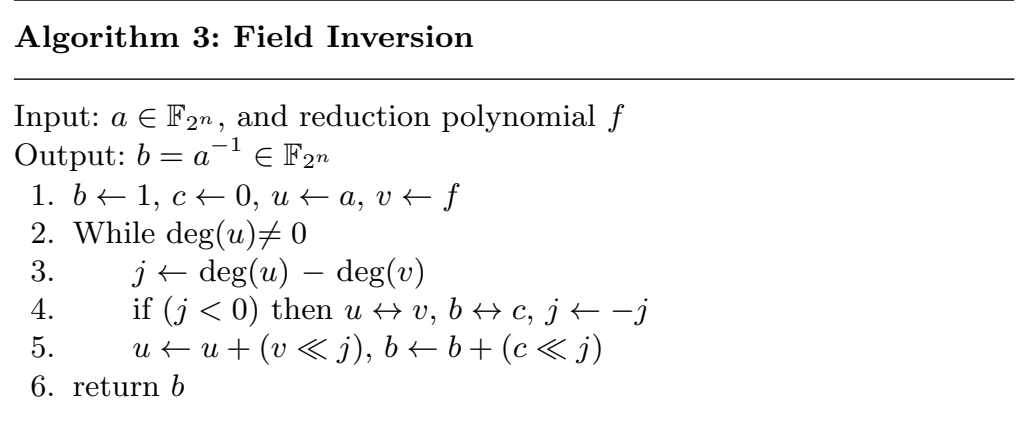

\subsection{Polynomial Rings}

The set of all polynomials with coefficients in $\mathbb{F}_{2^{n}}$ forms a ring, and is denoted $\mathbb{F}_{2^{n}}[u]$. This section discusses the mathematical aspects of dealing with these polynomials.

Ring Addition. Addition of two polynomials over a finite field equates to adding each term of each coefficient. That is, to add two polynomials of degree $m$, with $a_{i}, b_{i} \in \mathbb{F}_{2^{n}}$

$$
\sum_{i=0}^{m-1} a_{i} u^{i}+\sum_{i=0}^{m-1} b_{i} u^{i}=\sum_{i=0}^{m-1}\left(a_{i}+b_{i}\right) u^{i}
$$

where $a_{i}+b_{i}$ is field addition defined in Section 2.1.

Ring Squaring. Since a characteristic two finite field is being used, polynomial squaring has the same property as when performing field squaring, where all odd powers of $u$ have a coefficient of zero. The result is $b_{2 i}=a_{i}^{2}, \forall 0 \leq i \leq \operatorname{deg}(a)$ where $a_{i}^{2}$ is calculated as in Section 2.1.

Ring Multiplication. To multiply two ring elements, again defer to the gradeschool method. When multiplying a polynomial by a scalar in the field, multiply each term of the polynomial by the scalar. To multiply two polynomials, extend these steps to Algorithm 4.

Ring Division. When dividing two polynomials $a$ and $b$, a quotient $q$ and remainder $r$ are obtained, such that $a=q \times b+r$. The algorithm required to complete this is a slight modification of the Euclidean algorithm, and is presented in Algorithm 5. 


\section{Algorithm 4: Ring Multiplication}

Input: $a, b \in \mathbb{F}_{2^{n}}[u]$

Output: $c=a \times b \in \mathbb{F}_{2^{n}}[u]$

1. $c \leftarrow 0$

2. for $j$ from $\operatorname{deg}(a)$ downto 0

3. $c \leftarrow(c \ll 1)+a_{j} \times b$

4. return $c$

$\times$ is scalar multiplication

$\ll$ is polynomial coefficient shift

\section{Algorithm 5: Ring Division}

$$
\begin{aligned}
& \text { Input: } a, b \in \mathbb{F}_{2^{n}}[u] \\
& \text { Output: }(q, r) \in \mathbb{F}_{2^{n}}[u]: a=q \times b+r \\
& \text { 1. } i \leftarrow\left(b_{\operatorname{deg}(b)}\right)^{-1}, r=a \\
& \text { 2. for } j \text { from } \operatorname{deg}(a)-\operatorname{deg}(b) \text { downto } 0 \\
& \text { 3. } \quad f \leftarrow\left(r_{\operatorname{deg} r} \times i\right) \ll j \\
& \text { 4. } \quad t \leftarrow b \times f \\
& \text { 5. } \quad r \leftarrow r+t, q \leftarrow q+f \\
& \text { 6. return }(q, r)
\end{aligned}
$$

\section{$2.3 \quad$ Hyperelliptic Curves}

Hyperelliptic curves are a special class of algebraic curves. The following equation defines a genus $g$ hyperelliptic curve.

$$
v^{2}+H(u) v=F(u)
$$

where $F(u)$ is a monic polynomial of degree $2 g+1$ and $H(u)$ is a polynomial with degree at most $g$. In this implementation, the ground field is $\mathbb{F}_{2^{113}}$, and it uses the curve definition

$$
v^{2}+u v=u^{5}+u^{2}+1
$$

whence $g=2, F(u)=u^{5}+u^{2}+1$, and $H(u)=u$.

Divisors. Divisors are pairs $\operatorname{denoted} \operatorname{div}(A, B)$, where $A$ and $B$ are polynomials that satisfy the congruence

$$
B^{2}+H(u) B \equiv F(u)(\bmod A)
$$

Divisors are essentially points on the Jacobian of the hyperelliptic curve. Since these polynomials could have arbitrarily large degree and still satisfy the equation, the notion of a reduced divisor is needed. In a reduced divisor, the degree of 
$A$ is no greater than $g$, and the degree of $B$ is less than the degree of $A$. Cantor's Algorithm includes a method for reducing divisors.

Additionally, $\operatorname{div}(A, B)$ can be normalized by multiplying $A$ by $\alpha^{-1}$, where $\alpha$ is the leading coefficient of $A$. Using normalized divisors speeds up several portions of Cantor's Algorithm.

Jacobian. The Jacobian of a hyperelliptic curve is the set of all reduced divisors. This set is a group under the binary operation defined in the following section. The largest prime dividing the size of this group determines the overall security of the cryptosystem. For the implemented curve the largest prime dividing the size of the group is sixty-eight digits long. This prime is much larger than the current recommended value, and is therefore considered secure.

A more space efficient implementation could be achieved using different curves over smaller fields, however the curve and field combination implemented here is significantly more secure than other combinations of similar complexity. A forthcoming publication, 4, expands the ideas presented here, and examines performance results of genus two curves over other field sizes.

Cantor's Algorithm. Originally presented in [3], Cantor's Algorithm has been the keystone of all computation on Jacobians of hyperelliptic curves. In [1], Cantor's original algorithm was modified for compatability with binary fields. The Koblitz's version of Cantor's Algorithm is presented in Algorithm 6.

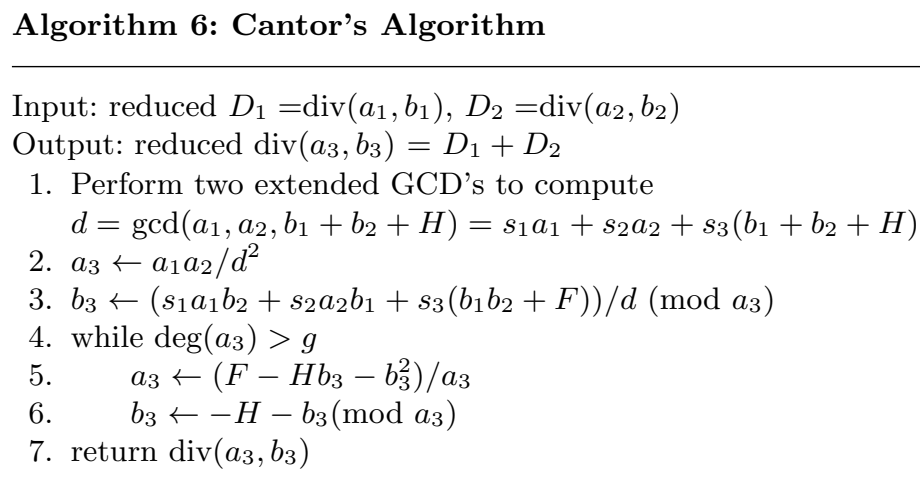

The algorithm can be broken down into three independent steps. The first step is computation of the extended GCD. The second is the composition step, corresponding to steps two and three above. Steps four through seven correspond to the reduction step. Improvements are discussed in each of the following sections. 
Table 1. Genus Two GCD Computation Cases

\begin{tabular}{|c|c|c|}
\hline & $d_{2} d_{3}$ & Result \\
\hline & $0-\infty$ & $d=1, s=(0,1,0)$ \\
\hline & $-\infty$ & $\begin{array}{l}\text { If } \gamma_{1}=\gamma_{2} \text { then } d=u-\gamma_{2}, s=(0,1,0) \\
\text { Else } d=1,\left(s_{1}, s_{2}\right)=L\left(a_{1}, a_{2}\right), s_{3}=0\end{array}$ \\
\hline 2 & $-\infty$ & $\begin{array}{l}\text { If } a_{1}\left(-\gamma_{2}\right)=0 \text { then } d=u-\gamma_{2}, s=(0,1,0) \\
\text { Else } d=1,\left(s_{1}, s_{2}\right)=M\left(a_{1}, a_{2}\right), s_{3}=0\end{array}$ \\
\hline 2 & $2-\infty$ & $\begin{array}{l}\text { Let } D=d \Delta_{b}-\Delta_{c}^{2} \text { where } d=\beta_{1} \gamma_{2}-\beta_{2} \gamma_{1}, \Delta_{b}=\beta_{2}-\beta_{1}, \Delta_{c}=\gamma_{2}-\gamma_{1} \\
\text { If } D \neq 0 \text { then } d=1,\left(s_{1}, s_{2}\right)=N\left(a_{1}, a_{2}\right), s_{3}=0 \\
\text { ElseIf } \beta_{1}=\beta_{2} \text { then } d=a_{1}, s_{1}=(0,1,0) \\
\text { Else } d=u+\Delta_{c} \Delta_{b}^{-1}, s=\left(-\Delta_{b}^{-1}, \Delta_{b}, 0\right)\end{array}$ \\
\hline & $\mathrm{x} 0$ & $d=1, s=(0,0,1)$ \\
\hline & 0 & $d=1, s=(0,1,0)$ \\
\hline & 1 & $\begin{array}{l}\text { If } \gamma_{1}=\gamma_{2}=\gamma_{3} \text { then } d=u+\gamma_{1}, s=(0,0,1) \\
\text { ElseIf } \gamma_{1} \neq \gamma_{2} \text { then } d=1, s_{3}=0,\left(s_{1}, s_{2}\right)=L\left(a_{1}, a_{2}\right) \\
\text { Else } d=1, s_{2}=0,\left(s_{1}, s_{3}\right)=L\left(a_{1}, a_{3}\right)\end{array}$ \\
\hline & 1 & $\begin{array}{l}\text { If } \gamma_{2} \neq \gamma_{3} \text { then } d=1, s_{1}=0,\left(s_{2}, s_{3}\right)=L\left(a_{2}, a_{3}\right) \\
\text { ElseIf } a_{1}\left(-\gamma_{2}\right) \neq 0 \text { then } d=1 . s_{1} \neq 0, s_{3}=0 \text { or } s_{2}=0 \\
\quad\left(s_{1}, s_{2}\right)=M\left(a_{1}, a_{2}\right) \text { or }\left(s_{1}, s_{3}\right)=M\left(a_{1}, a_{3}\right) \\
\text { Else } d=u+\gamma_{2}, s=(0,0,1)\end{array}$ \\
\hline & 2 & $\begin{array}{l}a_{3}=u+\gamma_{3} \\
\text { If } a_{1}\left(-\gamma_{3}\right)=a_{2}\left(-\gamma_{3}\right)=0 \text { then } d=u+\gamma_{3}, s=(0,0,1) \\
\text { ElseIf } a_{1}\left(-\gamma_{3}\right) \neq 0 \text { then } d=1, s_{2}=0,\left(s_{1}, s_{3}\right)=M\left(a_{1}, a_{3}\right) \\
\text { Else } d=1, s_{1}=0,\left(s_{2}, s_{3}\right)=M\left(a_{2}, a_{3}\right)\end{array}$ \\
\hline & $N\left(a_{1}\right.$ & $\begin{array}{c}L\left(a_{1}, a_{2}\right)=\left(-\left(\gamma_{2}-\gamma_{1}\right)^{-1}, \gamma_{2}-\gamma_{1}\right) \\
M\left(a_{1}, a_{2}\right)=\left(-a_{1}\left(-\gamma_{2}\right)^{-1}, a_{1}\left(-\gamma_{2}\right)^{-1} u+\left(\beta_{1}-\gamma_{2}\right) a_{1}\left(-\gamma_{2}\right)^{-1}\right) \\
\left.a_{2}\right)=\left(\left(\Delta_{b} / D\right) u+\left(\beta_{2} \Delta_{b}-\Delta_{c}\right) / D,\left(-\Delta_{b} / D\right) u+\left(-\beta_{1} \Delta_{b}+\Delta_{c}\right) / D\right)\end{array}$ \\
\hline
\end{tabular}

Extended GCD Calculation. Traditionally, the Extended Euclidean Algorithm (EEA) is used twice to calculate the greatest common divisor of three polynomials, resulting in $d=s_{1} a_{1}+s_{2} a_{2}+s_{3} a_{3}$ where $a_{3}=b_{1}+b_{2}+H$. However, for genus two curves with $\operatorname{deg}(H) \leq 1$, the degrees of $a_{1}, a_{2}$, and $a_{3}$ are maximally (and most frequently) 2,2 , and 1 , respectively. Since $a_{3}$ is of degree one, it cannot be factored. Hence, the GCD of the original three polynomials must be either 1 or $a_{3}$. Similar arguments can be made for the other possible degree cases. The complete proof of these results is not included in this paper, but will be available in [4.

Table 1 shows a list of all the different cases for the degrees of $a_{1}, a_{2}$, and $a_{3}=b_{1}+b_{2}+H$, and the explicit solution to the GCD and $s_{i}$ values. For the cases shown in Table $1, d_{i}=\operatorname{deg}\left(a_{i}\right), a_{1}(u)=u^{2}+\beta_{1} u+\gamma_{1}, a_{2}(u)=u^{2}+\beta_{2} u+\gamma_{2}$, and $a_{3}(u)=\beta_{3} u+\gamma_{3}$. Additionally, assume that if $\operatorname{deg}\left(a_{2}\right)>\operatorname{deg}\left(a_{1}\right), a_{1}$ and $a_{2}$ are swapped prior to computation.

Composition Step. The standard composition step in Cantor's Algorithm requires two polynomial divisions, in addition to a third in the form of modular 
reduction. In general, polynomial divisions are very time consuming in hardware. However, for genus two curves, notice that $d$ will almost always equal one. The only occurance of degree one is when $b_{1}+b_{2}+H$ exactly divides both $a_{1}$ and $a_{2}$. Therefore, most of the time the two polynomial divisions can be completely removed. Additionally, notice that four multiplications in the composition step can be completed in parallel with the GCD to further increase speed.

Reduction Step. Consider the following:

Proposition 1. For any hyperelliptic curve of genus $g$, at most $\left\lceil\frac{g}{2}\right\rceil$ reduction iterations are required to completely reduce any semi-reduced divisor.

Proof. See [15], proof 51.

Corollary 1. Semi-reduced divisors of a genus two curve require at most a single reduction iteration.

Given Corollary 1, the control logic for the reduction portion of Cantor's algorithm can be simplified. The while loop can be replaced with a single if block. In [18], Nigel Smart presents an alternate reduction algorithm, based on Tenner reduction. In a genus two case where only one reduction is required, this algorithm is identical to the standard algorithm.

\subsection{HEC Cryptosystems}

As with most public-key cryptosystems, HEC cryptosystems are usually only used for a symmetric key exchange, using the Diffie-Hellman protocol. Additionally, they can be used to sign messages using the Digital Signature Algorithm (DSA). For details on these algorithms, see [2].

Both algorithms involve scalar point multiplication (divisor $P$ multiplied by scalar integer $k$ ) on the Jacobian of the HEC, which is defined as adding $P$ to itself $k-1$ times. Since $k-1$ point additions is very slow, a square-and-add approach called binary expansion can be used. Given $k$ is expressed as a binary vector, the bases can be calculated by repeatedly doubling $P$. Then for each 1 in the binary representation of $k$, add the appropriate basis to a running total. On average, this requires $n$ doubles and $\frac{n}{2}$ adds, and can be efficiently implemented using a point doubler and a point adder operating in parallel.

A point doubler is essentially a special case of a point adder where both inputs are equal. Many multiplications can be replaced with squaring operations, which are significantly faster because field squaring can be implemented very quickly.

Given it takes $\alpha$ time units to complete an add, and $\beta$ time units to complete a double, the statistical expected amount of time required to multiply a point by an $n$-bit integer can be computed. For $\alpha<2 \beta$, the time required is discussed in Proposition 2. 
Proposition 2. Let $\Theta$ be an endomorphism of group $G$ (e.g. the doubling map for binary expansion). Let $k_{i}$ be statistically independent and not equaling 0 with probability $\delta ; \alpha$ be the time to perform group addition; and $\beta$ be the time required to compute $k_{i} \Theta^{i} P$ given $\Theta^{i-1} P$, with $\delta \alpha<\beta$ and the two devices operating in parallel. The expected time $T$ to compute

$$
\left(\sum_{i=0}^{n-1} k_{i} \Theta^{i}\right) P=\sum_{i=0}^{n-1} k_{i}\left(\Theta^{i} P\right)
$$

has a sharp upper bound of $\frac{\alpha^{2} \delta(1-\delta)}{2(\beta-\alpha \delta)}+\alpha \delta+(n-1) \beta$.

Proof. The overall system can be modeled as a discrete time D/G/1 queue (see [9]). Evaluation of the endomorphism corresponds to queue arrivals with deterministic interarrival times $X$ equal to $\beta$. For the service time process $Y$, each queue departure is nonzero with probability $\delta$ and hence has service time $\alpha$. The following are the first and second order statistics for stochastic processes $X$ and $Y$ :

$$
E[X]=\beta \quad \operatorname{Var}(X)=0 \quad E[Y]=\alpha \delta \quad \operatorname{Var}(Y)=\alpha^{2} \delta(1-\delta)
$$

For a reasonably large $n$, the mean system waiting time, $W$, in equilibrium converges in distribution to the mean system waiting time after $(n-1) \beta$ time units due to the basic principles of renewal theory (see [9]). Therefore, $T \stackrel{d}{=}$ $W+(n-1) \beta . W$ can be easily bounded above by the Kingman Moment Bound (see [1]).

$$
W \leq \frac{(1 / E[X])(\operatorname{Var}(X)+\operatorname{Var}(Y))}{2(1-E[Y] / E[X])}+E[Y]
$$

Substituting the statistics:

$$
T \stackrel{d}{=} W+(n-1) \beta \leq \frac{\frac{1}{\beta}\left(\alpha^{2} \delta(1-\delta)\right)}{2\left(1-\frac{\alpha \delta}{\beta}\right)}+\alpha \delta+(n-1) \beta=\frac{\alpha^{2} \delta(1-\delta)}{2(\beta-\alpha \delta)}+\alpha \delta+(n-1) \beta
$$

Notice that as $\alpha \delta$ approaches $\beta$, the bound on the processing time goes to infinity. This is because the queue length builds up indefinitely and the system is no longer positive recurrent; the waiting time after $(n-1) \beta$ time units cannot be approximated by the equilibrium distribution, and no general solution is possible using this model.

Corollary 2. In the case where $\theta=[2]$ and $G$ is the Jacobian of a hyperelliptic curve: $\delta=\frac{1}{2}, \alpha$ is the time to perform point addition, and $\beta$ is the time to complete point doubling, with $\alpha<2 \beta$. Therefore the mean time $T$ to perform a point multiplication has a sharp upper bound of $\frac{\alpha^{2}}{8 \beta-4 \alpha}+\frac{\alpha}{2}+(n-1) \beta$ 


\section{Coprocessor Implementation}

In order to effectively utilize FPGA area, the final coprocessor includes two polynomial multipliers and one each of the other polynomial computation blocks. A control unit is responsible for channeling data in and out of the computation blocks, implementing Cantor's algorithm.

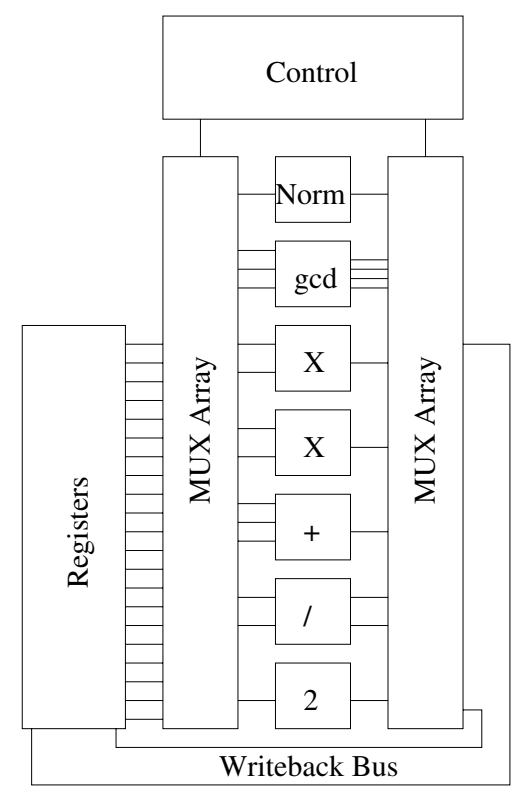

Fig. 1. Overall Coprocessor Architecture

\subsection{Field Calculation Blocks}

Since field addition is such a simple operation, a separate Verilog module was not created to implement it.

Field multiplication is at the heart of most calculations, and therefore must be done quickly and efficiently. Implemented in hardware, Algorithm 1 uses combinatorial logic to compute $((c+a) \ll 1)+f,(c+a) \ll 1,(c \ll 1)+f$, and $c \ll 1$ and a multiplexor to select the correct result for a given iteration.

While an alternate multiplication design which processes multiple bits at a time could be implemented, effectively making the coprocessor operate two to three times faster, the extra area requirements are not physically realizable on a Xilinx FPGA. These are known as $D=X$ multipliers where $X$ is the number of bits that can be simultaneously computed.

For field squaring, Algorithm 2 step two is a rewiring, using no gates. If the upper bits are zero (such as when squaring 1), the algorithm is complete. 
Otherwise, during each loop iteration, updating $b$ and $g$ can occur independently and consequently during the same clock cycle. By considering two bits of $g$ rather than one, steps five and six can be combined using a multiplexor.

Field inversion is used in the polynomial GCD and polynomial division blocks. Algorithm 3 is implemented as a finite state machine.

Table 2. Field Implementation Results

\begin{tabular}{|c|c|c|c|}
\hline Module & Clock Cycles & Slices & Max Speed \\
\hline \hline Field Multiplication & 2 or $115^{a}$ & 399 & $96 \mathrm{MHz}$ \\
\hline Field Squaring & 2 or $59^{b}$ & 186 & $124 \mathrm{MHz}$ \\
\hline Field Inversion & $395($ avg) & 1631 & $98 \mathrm{MHz}$ \\
\hline
\end{tabular}

${ }^{a}$ When multiplying by zero or one, the result is immediate

${ }^{b}$ When squaring a field element of degree $\left\lfloor\frac{n}{2}\right\rfloor$ or less, only reassignment is required

\subsection{Polynomial Calculation Blocks}

Polynomial blocks are collections of the appropriate field blocks combined to implement various algorithms. The control structure only has access to polynomial units; therefore, all functionality required by Cantor's Algorithm must be available through polynomial calculation blocks. Additionally, polynomials have no maximum degree. The input size for each polynomial block was determined by the maximal value of the intermediate states, and ensures there will be no overflow problems.

The addition block accepts three input polynomials of maximum degree six, and returns their sum. This requires 1582 XOR gates and is completely combinatorial.

The maximum degree ever encountered during multiplication for the first input is five, and the second input is two. Therefore, the polynomial multiplier must accommodate multiplication of a degree five polynomial by a degree two polynomial. This requires each of six field multipliers to complete three multiplications, as demonstrated by Algorithm 4. The version implemented in this design includes cases to check for multiplication by both zero and one, which results in a two clock-cycle calculation.

The ring squarer accepts inputs with degree up to three, and returns polynomials of maximal degree six. Its implementation consists of four field squarers, operating in parallel.

Ring division is by far the most complex and time-consuming operation. The number of required clock cycles greatly depends on the degrees of the two input polynomials, which can range from zero to six for the numerator, and zero to four for the denominator. The greater the difference in degree, the longer 
the processing takes. A special case of scalar multiplication by the inverse was incorporated for a zero degree denominator.

Ring normalization makes a degree two polynomial monic by multiplying its terms by the inverse of its leading coefficient. It is implemented using a field inverter and two field multipliers.

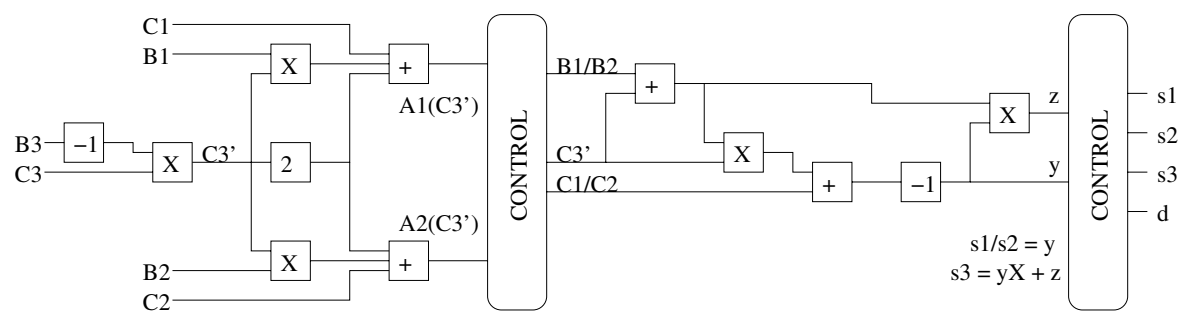

Fig. 2. Dual Degree Two Case GCD Calculation Block

While all the cases described in Table 2 are significantly faster than using the Extended Euclidean Algorithm for the GCD computations, the chip area required to implementation all the cases is prohibitive. Therefore, only the final case where $d_{1}=d_{2}=2$ is implemented as shown in Figure 2, using two field multipliers, a field squarer, and a field inverter. For all other cases, the coprocessor control subsystem uses the already existing polynomial logic blocks to perform the Extended Euclidean Algorithm. Since the probability of having $d_{1}<2$ or $d_{2}<2$ is small, the adverse performance effects are trivial.

Table 3. Ring Implementation Results

\begin{tabular}{|c|c|c|c|}
\hline Module & Clock Cycles & Slices & Max Speed \\
\hline \hline Ring Addition & 1 & 791 & $83 \mathrm{MHz}$ \\
\hline Ring Multiplication & 2 to 353 & 1,561 & $64 \mathrm{MHz}$ \\
\hline Ring Squaring & 2 or 59 & 515 & $55 \mathrm{MHz}$ \\
\hline Ring Division & 2 to 2,300 & 8,337 & $80 \mathrm{MHz}$ \\
\hline Ring GCD & $1,270(\mathrm{avg})$ & 3,515 & $96 \mathrm{MHz}$ \\
\hline Ring Norm & $615(\mathrm{avg})$ & 2,488 & $71 \mathrm{MHz}$ \\
\hline
\end{tabular}

\subsection{Control Implementation}

Using the available polynomial computation blocks, a control block operated by a finite state machine decides which data should enter each polynomial ring unit, decides when the unit is done processing, and keeps track of intermediate variables as Cantor's algorithm progresses. The scheduling is programmed into 
the state machine. Since some computation blocks require a variable number of clock cycles to complete, the control mechanism does not try to use a result that has not yet been obtained, and it also does not waste time waiting for a result that has already returned.

Two different control mechanisms were designed. The first is a finite state machine which performs general point addition. The second is a finite state machine which performs point doubling. The point doubler is not as simple as the one described in [18] because the implemented curve uses $H(u)=u$ rather than $H(u)=1$. The implementation results of both are included in the next section.

\section{Performance}

Since the emphasis of this paper is on the hardware implementation, the software implementation is not extensively discussed. The original intent of the software version was to verify the correctness of the hardware version; however, an effort was made to minimize both memory usage and runtime. On a Pentium III, 1.2 $\mathrm{GHz}$, point addition, doubling, and multiplication can be completed in 1.97, 1.01, and 222 milliseconds, respectively.

Using the Xilinx ISE tools with target chip configured as the Virtex II FPGA, the final implementation results for the hardware version are stated in Table 4. Through both experimental tests and application of Corollary 2, point multiplication can be achieved in 10.1 milliseconds. The smallest Xilinx device supporting the full point multiplication architecture using a parallel adder and doubler is the Virtex II 2VP30, which has 30,816 slices.

Table 4. Hardware Implementation Results

\begin{tabular}{|c|c|c|c|c|}
\hline Operation & Clock Cycles & Slices & Max Speed & Time \\
\hline \hline Point Addition & 4,750 & 16,600 & $45 \mathrm{MHz}$ & $105 \mu \mathrm{s}$ \\
\hline Point Doubling & 4,050 & 15,100 & $45 \mathrm{MHz}$ & $90 \mu \mathrm{s}$ \\
\hline
\end{tabular}

\section{Conclusion}

By using many standard finite field algorithms, and an alternate method for computing the GCD, this implementation is proof that HECC can be implemented at speeds comparable to ECC, and realizable in areas suited for embedded applications.

An important thing to keep in mind when comparing these results to those of ECC coprocessors implementing an equal level of security is the algorithm complexity. Cantor's algorithm operates over polynomials and not individual field elements, which results in much higher FPGA area requirements. The timing 
results in Table 4 could easily be reduced by a factor of 10 by using a $D=16$ field multiplier (such as in [16]); however, such a chip would have unreasonable space requirements and not be physically realizable on most FPGA's.

Future work on this project is expected at both the hardware and software levels. We are interested in carrying the implementation forward and completing the ASIC level design and eventual fabrication of the chip. Additionally, we are extending the implementation to use the $\tau$-adic method for point multiplication, as described in [8] and more extensively in [13]. We are also examining implementations of a genus three curve over $\mathbb{F}_{2^{61}}$, on a 64 -bit system.

\section{References}

1. D. Bertsekas, R. Gallager. Data Networks. Prentice Hall: New Jersey. 1992.

2. I. Blake, G. Seroussi, N. Smart. Elliptic Curves in Cryptography. London Mathematical Society Lecture Notes Series, Volume 265. University Press: Cambridge. 1999.

3. D. Cantor. "Computing the Jacobian of a Hyperelliptic Curve." Mathematics of Computation, v48, pp 95-101. January 1987.

4. T. Clancy, Y. Liow. "FPGA-based Hyperelliptic Curve Cryptosystems." to appear.

5. D. Dummit, R. Foote. Abstract Algebra. John Wiley and Sons: New York. 1999.

6. D. Hankerson, J. Hernandez, A. Menezes. "Software Implementation of Elliptic Curve Cryptosystems Over Binary Fields." CHES 2000, LNCS, Volume 1965, pp 1-24. Springer-Verlag: New York. 2000.

7. T. Hungerford. Algebra. Graduate Texts in Mathematics, Volume 73. SpringerVerlag: New York. 1974.

8. C. Gunther, T. Lange, A. Stein. "Speeding up the Arithmetic on Koblitz Curves of Genus Two." SAC 2000, LNCS, Volume 2012, pp 106-117. Springer-Verlag: New York. 2001.

9. L. Kleinrock. Queueing Systems, Volume I: Theory. John Wiley and Sons: New York. 1975.

10. N. Koblitz. "A Family of Jacobians Suitible for Discrete Log Cryptosystems." CRYPTO 1988, LNCS, Voume 403, pp 94-99. Springer-Verlag: New York. 1988.

11. N. Koblitz. "Hyperelliptic Cryptosystems." Journal of Cryptology, Volume 1, pp 139-150. 1989.

12. N. Koblitz. Algebraic Aspects of Cryptography. Algorithms and Computation in Mathematics, Volume 3. Springer: New York. 1998.

13. T. Lange. "Fast Arithmetic on Hyperelliptic Curves." PhD Thesis, Institute for Information Security and Cryptography, Ruhr-Universität Bochum. 2002.

14. A. Menezes, P. van Oorschot, S. Vanstone. Handbook of Applied Cryptography. CRC Press. 1997.

15. A. Menezes, Y. Wu, R. Zuccherato. "An Elementary Introduction to Hyperelliptic Curves." appendix in Algebraic Aspects of Cryptography N. Koblitz, SpringerVerlag, 1998.

16. G. Orlando, C. Paar. "A High Performance Reconfigurable Elliptic Curve Processor for $\operatorname{GF}\left(2^{m}\right)$." CHES 2000, LNCS, Volume 1965, pp 41-56. Springer-Verlag: New York. 2000.

17. J. Silverman. Arithmetic of Elliptic Curves. Graduate Texts in Mathematics, Volume 106. Springer-Verlag: New York. 1986. 
$414 \quad$ N. Boston et al.

18. N. Smart. "On the Performance of Hyperelliptic Cryptosystems." HPL-98-162. HP Extended Enterprise Laboratory. September 1998.

19. L. Song, K. Parhi, "Low-Energy Digit-Serial/Parallel Finite Field Multipliers," Journal of VHDL Signal Processing Systems. pp 1-17. 1997.

20. T. Wollinger. "Computer Architectures for Cryptosystems Based on Hyperelliptic Curves." Master's Thesis, Worcester Polytechnic Institute. April 2001. 